\title{
Modelling of EUV light sources based on microwave discharge in inhomogeneous flow of nonequilibrium plasma with multiply charged tin and xenon ions
}

\author{
I. S. Abramov, E. D. Gospodchikov, A. G. Shalashov \\ Institute of Applied Physics of Russian Academy of Sciences, Nizhny Novgorod, Russia, abramov@appl.sci-nnov.ru
}

\section{Introduction}

Extreme ultraviolet (EUV) lithography is key technology for further decrease of integral circuit (IC) scales [1]. Transition to smaller wavelength of exposing radiation requires generation of plasma with multiply charged ions of heavy elements emitting EUV light. Now IC manufacturers use sources based on laser evaporation of tin droplets that can provide $200-250 \mathrm{~W}$ of EUV light in $13.5 \mathrm{~nm} \pm 1 \%$ spectral band [2-4]. However experts believe that for significant improvement of IC form factor about $1 \mathrm{~kW}$ power is required that can hardly be obtained in laser produced plasmas [4].

A competitive alternative is microwave discharge supported by submillimeter wave radiation of modern high power gyrotrons. Duration of gyrotron pulses is much longer (50 $\mu$ s to $\mathrm{CW})$ and provided power is much higher (up to $1 \mathrm{MW}$ ) than those for lasers in use. These allow to support strongly radiating discharge in stationary mode. Moreover, microwave heating leads to nonequilibrium plasma with highenergy electrons and as a consequence high rates of electron impact ionization and excitation. Two concepts of such EUV light sources were developed in IAP: based on tin plasma flow propagating through the open magnetic trap and freely expanding flow of xenon plasma $[5,6]$. Both concepts are tested in proof of principle experiments, but detailed theoretical study is required to explore physical constrains and prospects for these concepts. This study is in focus of present communication.

\section{Theory}

We use fluid equations taking into account multiple ionization by electron impact with the following assumptions:

- the discharge is stationary, thus we use stationary equations;

- the plasma is strongly inhomogeneous along the flow direction $x$, thus we assume plasma flow with the characteristics averaged over the flow cross section $S(x)$;

- difference in ion and electron masses allows to neglect the electron momentum flow;

- the temperature of electrons is much higher than ion one $T_{e} \gg T_{i}$ due to resonant heating, thus ion pressure is neglected;

- high electron temperature provides high electron thermal conductivity, thus $T_{e}$ is conserved and enters the model as a parameter.

Under the assumptions above, fluid equations may be expressed as ordinary differential equations of ion fractions particle balance and the equation of total momentum balance $[7,8]$

$$
\begin{gathered}
\frac{d}{d x}\left(n_{j} S u\right)=n_{e} S\left(k_{j-1} n_{j-1}-k_{j} n_{j}\right), \\
\frac{d}{d x}\left(S M n u^{2}\right)+S \frac{d}{d x}\left(n_{e} T_{e}\right)=0, n=\sum_{j=1}^{Z_{\max }} n_{j},
\end{gathered}
$$

where $n_{j}$ are densities of ions with charge-state $Z_{j}=j$ (i.e. with charge $j e), u$ is the flow velocity, $n_{e}$ is the electron density followed from the quasi-neutrality of the plasma, $k_{j}$ is $j$-th ionization constant, $M$ is ion mass. As it follows from (1), the total flow $F=S n u$ is conserved and may be treated as another model parameter. Thus to close the problem it is necessary to set ion composition and flow velocity at some point inside the discharge. Boundary conditions for (1) and (2) are set taking into account presence of ion acoustic transition in the flow expanding into vacuum [8]. Formal mathematical difficulties with defining the transition location are treated as it is proposed in [9].

From the energy conservation, a power load that is required to support the high electron temperature may be estimated as sum over major channels of power losses from the discharge

$$
P=\left.A F \frac{M u^{2}}{2}\right|_{x \rightarrow L}+\int\left(p_{\text {ion }}+p_{\text {rad }}\right) S d x .
$$

The first term represents the convective losses; here $A$ is the coefficient depending on details of the plasma absorber located at $L[8]$. The second term describes the volumetric losses for ionization and line radiation of ions characterized with densities

$$
p_{\text {ion }}=\sum_{j} E_{j} k_{j} n_{e} n_{j}, \quad p_{\text {rad }}=\sum_{j} \sum_{l} \sum_{h} E_{j h l} k_{j l h}^{*} n_{e} n_{j} .
$$

Here $p_{\text {ion }}$ is the power spent to ionize a unit volume of multiply charged plasma, $E_{j}$ is the ionization energy of $j$-th ion, $k_{j} n_{e}$ is the effective rate of electron impact ionization, $p_{\text {rad }}$ is the power spent on line radiation and inner sums are taken over all possible terms of ground $(l)$ and excited $(h)$ electron configurations of $j$-th ion, $E_{j h l}$ are the transition energies. Values $k_{j l h}^{*}$ are effective excitation constants. Plasmas with densities close to $10^{16} \mathrm{~cm}^{-3}$ that may be obtained in studied discharges are not optically thin even for energetic EUV light quanta. So, for these conditions the constants $k_{j l h}^{*}$ should be modified compared to optically thin limit by taking into account the effect of radiation trapping due to reabsorption of the emitted photons in the discharge volume. The method is developed by the authors and discussed in details in [10].

The theory was used to study prospects of both concepts of EUV light sources proposed in IAP. Here is the brief overview of the results. 


\section{Tin plasma in open magnetic trap}

The first concept of the source based on additional heating of the vacuum arc plasma flow propagating in open magnetic configuration shown possibilities to generate large amounts of EUV power up to tens of kilowatts at present level of technological development (with modern gyrotrons and vacuum arc sources). However the main problem of these sources is that the emitting region is pretty long and it is nearly impossible to collect and focus all generated EUV light. Even modified magnetic configuration proposed by authors in [9] that significantly decreases the emitting area hardly can provide required sizes of about $1 \mathrm{~mm}$. Fig. 1 illustrates modeling of the EUV light source that provides about $40 \mathrm{~kW}$ of the required radiation with about $20 \%$ of microwave to EUV conversion efficiency. Emitting region size is about $1 \mathrm{~cm}$.
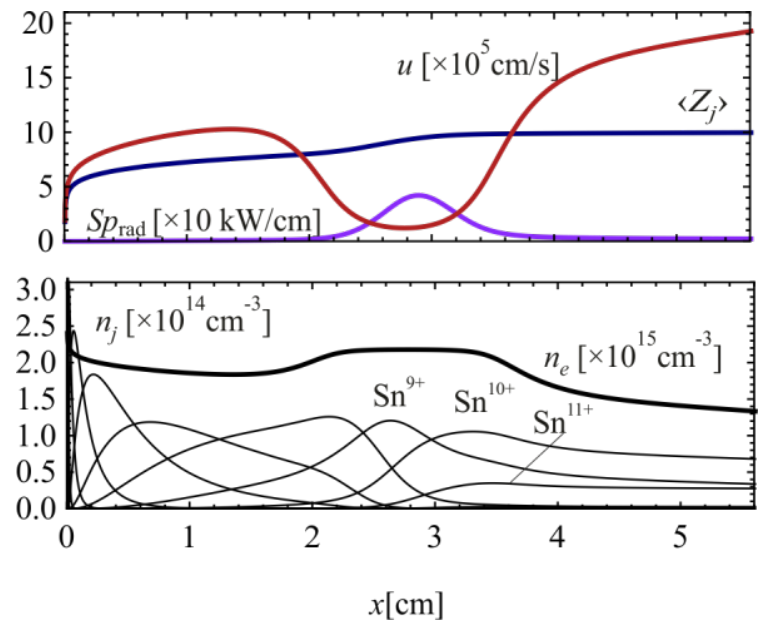

Fig. 1. Simulation of EUV-radiating discharge in tin plasma flow propagating through the open magnetic trap: variation along the trap axis of the flow velocity $u$ (red line, top panel), average ion charge $\left\langle Z_{j}\right\rangle$ (blue line), linear power density $S p_{\text {rad }}$ of radiation in $13.5 \pm 1 \% \mathrm{~nm}$ EUV band (violet line), densities of ion fractions $n_{j}$ (thin black lines, bottom panel), and electron density $n_{e}$ (thick black line). Model parameters are: trap length $L=5.6 \mathrm{~cm}$, mirror ratio $R=9$, initial plasma diameter $d=0.2 \mathrm{~cm}$, arc current $I_{a}=2.9 \mathrm{kA}$, electron temperature $T_{e}=50 \mathrm{eV}$, initial beam composition $n_{1}(0) / n(0) \approx 0.325, n_{2}(0) / n(0) \approx 0.567, n_{3}(0) / n(0) \approx 0.108$, and other components are negligible.

\section{Discharge in freely expanding xenon jet}

The second concept involves microwave discharge in freely expanding jet of heavy noble gases (presumably xenon). Such jet provides perfect (less than $1 \mathrm{~mm}$ ) localization of the discharge but causes decrease in conversion efficiency due to increasing percentage of convective losses. To provide EUV light power of $\mathrm{kW}$ level by such a small discharge dense plasmas are required. However it is shown that gas puff rate and heating may be adjusted so that radiation trapping becomes a positive effect as more energetic radiation quanta are trapped at higher densities. Fig. 2 illustrates modeling of the EUV light source that provides about $5 \mathrm{~kW}$ of the required radiation with about $5 \%$ of microwave to EUV conversion efficiency. Emitting region size is smaller than $1 \mathrm{~mm}$.
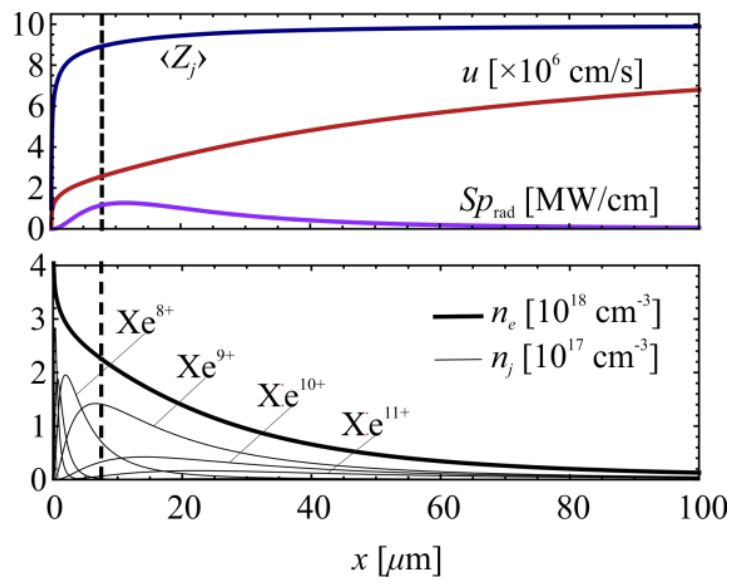

Fig. 2. Simulation of EUV-radiating discharge in freely expanding xenon jet: flow velocity $u$ (red line, top panel, $x$ is the distance from the nozzle), average ion charge $\left\langle Z_{j}\right\rangle$ (blue line), linear power density $S p_{\text {rad }}$ of radiation in $11.2 \pm 1 \% \mathrm{~nm}$ EUV band (violet line), densities of ion fractions $n_{j}$ (thin black lines, bottom panel), and electron density $n_{e}$ (thick black line). Parameters: nozzle diameter $d=70 \mu \mathrm{m}$, total flow $F=2 \times 10^{19} \mathrm{~s}^{-1}$, electron temperature $T_{e}=100 \mathrm{eV}$. Xenon is singly ionized at the nozzle.

\section{Acknowledgements}

The work was supported by Russian Foundation for Basic Research (Grants No. 17-02-00173 and No. 18-32-00419).

\section{References}

1. Wagner, C., Harned, N. Lithography gets extreme // Nature Photonics 2010. V. 4, P. 24-26.

2. Mizoguchi, H., Nakarai, H., Abe, T. et al. Performance of 250W high-power HVM LPP-EUV source // EUV Lithography VIII 2017, V. 10143. P. 101431J.

3. Fomenkov, I., Brandt, D., Ershov, A. et al. Light sources for high-volume manufacturing EUV lithography: technology, performance, and power scaling // Advanced Optical Technologies 2017, V. 6, P. $173-186$.

4. Moore, S. EUV lithography finally ready for fabs // IEEE Spectrum 2018, V. 55. P. 46-48.

5. Chkhalo, N. I., Golubev, S. V. et al. Source for extreme ultraviolet lithography based on plasma sustained by millimeter-wave gyrotron radiation // J. Micro Nanolithogr. MEMS and MOEMS 2012, V. 11, P. 021123.

6. Glyavin, M. Y., Golubev, S. V., Izotov, I. V. et al. A point-like source of extreme ultraviolet radiation based on a discharge in a non-uniform gas flow, sustained by powerful gyrotron radiation of terahertz frequency band // Applied Physics Letters 2014, V.105(17), P. 174101.

7. Abramov, I. S., Gospodchikov, E. D., Shalash$o v, A$. G. Formation of a multi-charged plasma in the directed gas flow // Radiophysics and Quantum Electronics 2016, V. 58. P. $914-933$.

8. Shalashov, A. G., Abramov, I. S., Golubev, S. V., Gospodchikov, E. D. Theory of a stationary microwave discharge with multiply charged ions in an expanding gas jet // JETP 2016, V. 123, P. 219 - 230.

9. Abramov, I. S., Gospodchikov, E. D., Shalashov, A. G. Prospects of extreme ultraviolet radiation sources based on microwave discharge for high-resolution lithography // Physics of Plasmas 2017, V. 24, P. 073511.

10. Abramov, I. S., Gospodchikov, E. D., Shalashov, A.G. Potentials of EUV light source based on microwave discharge in expanding jet of dense xenon plasma // arXiv:1712.10026 [physics.plasm-ph] 2018. 